\title{
Consistent frequency-matching calibration procedure for electromechanical shunt absorbers
}

\section{Høgsberg, Jan}

Published in:

Journal of Vibration and Control

Link to article, DOI:

$10.1177 / 1077546320924207$

Publication date:

2020

Document Version

Peer reviewed version

Link back to DTU Orbit

Citation (APA):

Høgsberg, J. (2020). Consistent frequency-matching calibration procedure for electromechanical shunt absorbers. Journal of Vibration and Control, 26(13-14), 1133-1144. https://doi.org/10.1177/1077546320924207

\section{General rights}

Copyright and moral rights for the publications made accessible in the public portal are retained by the authors and/or other copyright owners and it is a condition of accessing publications that users recognise and abide by the legal requirements associated with these rights.

- Users may download and print one copy of any publication from the public portal for the purpose of private study or research.

- You may not further distribute the material or use it for any profit-making activity or commercial gain

- You may freely distribute the URL identifying the publication in the public portal

If you believe that this document breaches copyright please contact us providing details, and we will remove access to the work immediately and investigate your claim 


\title{
CONSISTENT FREQUENCY-MATCHING CALIBRATION PROCEDURE FOR ELECTROMECHANICAL SHUNT ABSORBERS
}

\author{
JAN HøGSBERG \\ Department of Mechanical Engineering, Technical University of Denmark, \\ Nils Koppels Allé, building 403, DK-2800 Kongens Lyngby, Denmark \\ email: jhg@mek.dtu.dk, ORCID: 0000-0002-1098-3245.
}

\section{INTRODUCTION}

Electromechanical transducers may be installed in structures to mitigate dynamic response (Yan et al., 2017). Especially piezoelectric transducers have been widely considered for vibration damping (Preumont, 2011), for example with supplemental shunts that dissipate energy in resistors (Moheimani and Fleming, 2006). Inductive-resistive $(R L)$ shunts further contain an inductance that creates a system resonance, which must be precisely synchronized with the targeted structure resonance to achieve superior performance. The concept of $R L$ shunt damping was initially proposed by Forward (1979) and subsequently propelled by Hagood and von Flotow (1991), who rigorously analyzed and calibrated a series $R L$ shunt. The alternative parallel $R L$ shunt was later considered by $\mathrm{Wu}$ (1996). Various calibration strategies have recently been derived based on different optimization principles (Caruso, 2001; Yamada et al., 2010; Høgsberg and Krenk, 2012). The performance of an $R L$ shunt is sensitive to mistuning (Berardengo et al., 2016, a), partly due to unaccounted for interaction with non-resonant residual modes (Høgsberg and Krenk, 2015). This modal coupling has been explicitly represented by Krenk and Høgsberg (2016) and subsequently used to improve the calibration formulae for piezoelectric $R L$ shunts (Høgsberg and Krenk, 2017). The electromechanical coupling in electromagnetic transducers may as well be used for $R C$ shunt damping (Behrens et al., 2003; Fleming, 2004; Behrens et al., 2005), with the analogy to piezoelectric $R L$ shunt damping addressed by Fleming (2004); Niederberger (2005); Scheidler and Asnani (2017); Høgsberg (2019). The $R C$ shunt components can for example be calibrated using the classic fixed-point principle of Den Hartog (1956), with various resistor calibrations proposed by Inoue et al. (2008); Zhu et al. (2013). Several calibration principles have been summarized by Tang et al. (2016) and realized in non-contact concepts (Cheng and Oh, 2009), in mechanical absorbers with an auxiliary mass (Fleming and Moheimani, 2006; Yan et al., 2016) or in adaptively controlled shunts (Niederberger et al., 2006). Optimal calibration of $R C$ shunts might require negative resistance to reduce the intrinsic coil resistance Niu et al. (2009), while a supplemental inductance has been suggested by Gonzalez-Buelga et al. (2015) to obtain improved performance outside the narrow resonance band. Finally, Electromagnetic transducers are scalable in size and potentially feasible for civil engineering scale structures (Palomera-Arias, 2005; Palomera-Arias et al., 2008).

A mechanical analogy with a spring, dashpot and inerter placed in series is derived for the electromagnetic series $R C$ shunt and piezoelectric parallel $R L$ shunt which directly includes intrinsic 
transducer resistance. The present paper hereby shows that the former can be calibrated explicitly by principles obtained for the latter, and vice versa. A property commonly ignored in the literature, as e.g. Inoue et al. (2008); Zhu et al. (2013) derive similar expressions for the electromagnetic shunt frequency without just referring to the results for the piezoelectric equivalence obtained earlier by $\mathrm{Wu}(1996)$. The present calibration procedure accounts for the influence from residual vibration modes by an augmented modal representation (Krenk and Høgsberg, 2016) with two correction terms. The corresponding correction coefficients can be consistently determined by comparing the two roots of the modal characteristic equation with the natural frequencies of the system with rigid absorber dashpots. This novel approach therefore avoids the matrix inversions used in previous studies and enables calibration of residual mode effects by measured frequencies. As demonstrated in the numerical example the proposed procedure therefore robustly recovers the desired modal damping ratios for an unsupported truss structure with six zero-frequency rigid body modes. A simplified quasi-static residual mode correction is related to a modal electromechanical coupling coefficient, which for the floating truss structure yields an almost insignificant reduction in attainable damping. The numerical example finally shows that without residual mode correction, the attainable damping by the resonant shunt absorbers is substantially reduced.

\section{Electromechanical systems}

In Fig. 1(a) the electromechanical coupling of an electromagnetic transducer produces a mechanical force $f$ when the magnetic core moves with velocity amplitude $i \omega x$ inside a conductive coil, with $x$ denoting the transducer deformation associated with the temporal exponential factor $\exp (i \omega t)$, in which $i=\sqrt{-1}$ is the imaginary unit, $\omega$ is the angular frequency and $t$ is time. For the piezoelectric transducer in Fig. 1(b) the energy conversion is generated from the straining $x$ of the solid actuator by the force $f$. A common absorber format is derived for the shunted electromagnetic and piezoelectric transducers, explicitly embracing intrinsic transducer effects and the residual mode correction consider in a subsequent section.

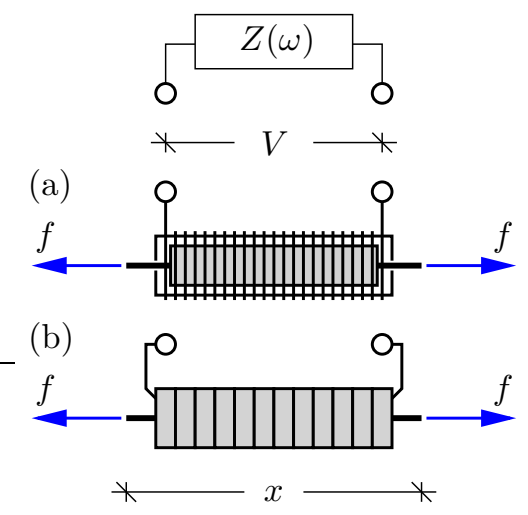

FiguRE 1. (a) Electromagnetic and (b) piezoelectric transducers with shunt $Z(\omega)$. 


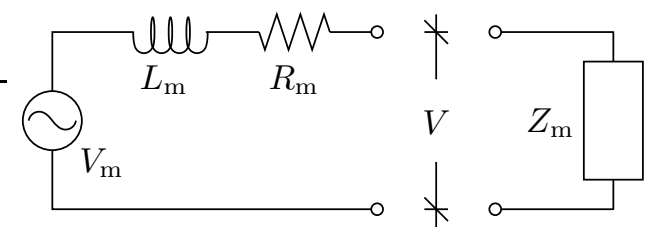

FiguRE 2. Electric network representing an electromagnetic transducer with shunt $Z_{\mathrm{m}}(\omega)$.

2.1. Electromagnetic shunt damping. For the electromagnetic transducer in Fig. 1(a) the force $f$ is assumed proportional to the coil current $I$,

$$
f=K_{\mathrm{m}} I
$$

introducing the coupling coefficient or motor constant $K_{\mathrm{m}}$ (Fleming, 2004). Figure 2 shows the electric representation of the shunted electromagnetic transducer in Fig. 1(a), in which the amplitude of the alternating voltage source

$$
V_{\mathrm{m}}=i \omega K_{\mathrm{m}} x
$$

is proportional to the velocity amplitude $i \omega x$ of the structure across the transducer terminals. For shorted terminals $I$ is governed by its inherent impedance with inductance $L_{\mathrm{m}}$ and resistance $R_{\mathrm{m}}$ (Palomera-Arias, 2005),

$$
V_{\mathrm{m}}=\left(R_{\mathrm{m}}+i \omega L_{\mathrm{m}}\right) I+V=i \omega K_{\mathrm{m}} x
$$

as shown in Fig. 2. Vibration damping is introduced by a shunt with impedance $Z=Z_{\mathrm{m}}$, defined by $V=Z_{\mathrm{m}}(\omega) I$ and used to eliminate the voltage $V$ in (3), that is then substituted back into (2) to give

$$
i \omega K_{\mathrm{m}} x=\left(R_{\mathrm{m}}+i \omega L_{\mathrm{m}}+Z_{\mathrm{m}}(\omega)\right) I
$$

Substitution of $I$ from (1) then defines the apparent mechanical flexibility relation

$$
x=\left(\frac{L_{\mathrm{m}}}{K_{\mathrm{m}}^{2}}+\frac{R_{\mathrm{m}}}{i \omega K_{\mathrm{m}}^{2}}+\frac{Z_{\mathrm{m}}(\omega)}{i \omega K_{\mathrm{m}}^{2}}\right) f
$$

which can be written as

$$
x=\left(\frac{1}{k_{\mathrm{m}}}+\frac{1}{i \omega c_{\mathrm{m}}}+\frac{1}{i \omega h_{\mathrm{m}}(\omega)}\right) f
$$

This represents the series mechanical model in Fig. 3(a) with a spring $k_{\mathrm{m}}$, viscous dashpot $c_{\mathrm{m}}$ and frequency dependent viscous dashpot $h_{\mathrm{m}}$ introduced as

$$
k_{\mathrm{m}}=\frac{K_{\mathrm{m}}^{2}}{L_{\mathrm{m}}}, \quad c_{\mathrm{m}}=\frac{K_{\mathrm{m}}^{2}}{R_{\mathrm{m}}}, \quad h_{\mathrm{m}}(\omega)=\frac{K_{\mathrm{m}}^{2}}{Z_{\mathrm{m}}(\omega)}
$$

The impedance $Z_{\mathrm{m}}(\omega)$ is subsequently chosen to exactly recover a desired mechanical vibration absorber format.

2.2. Piezoelectric shunt damping. For the piezoelectric transducer in Fig. 1(b), the force $f=K_{\mathrm{p}} V$ is directly proportional to the applied voltage $V$ between the transducer electrodes, with $K_{\mathrm{p}}$ being the electromechanical coupling coefficient. The electrical loading from straining of the transducer is represented by the alternating current source $I_{\mathrm{p}}=i \omega K_{\mathrm{p}} x$, placed in parallel with the inherent transducer capacitance $C_{\mathrm{p}}$ and a large equivalent resistance $R_{\mathrm{p}}$ (often neglected), 
representing hysteresis (Forward, 1979). Thus, the piezoelectric transducer is described by the network in Fig. 4, for which Kirchhoff's current law gives

$$
I_{\mathrm{p}}=\left(i \omega C_{\mathrm{p}}+\frac{1}{R_{\mathrm{p}}}\right) V+I=i \omega K_{\mathrm{p}} x
$$

with $I$ being the current through the electrodes. The current $I$ is eliminated by the impedance relation $V=Z_{\mathrm{p}}(\omega) I$, while voltage is subsequently replaced by $V=f / K_{\mathrm{p}}$, thus defining an apparent mechanical relation

$$
x=\left(\frac{1}{k_{\mathrm{p}}}+\frac{1}{i \omega c_{\mathrm{p}}}+\frac{1}{i \omega h_{\mathrm{p}}(\omega)}\right) f
$$

that is identical to that in Fig. 3(a) when ' $m$ ' is replaced by 'p'. For the piezoelectric transducer the equivalent mechanical components are

$$
k_{\mathrm{p}}=K_{\mathrm{p}}^{2} C_{\mathrm{p}}^{-1}, \quad c_{\mathrm{p}}=K_{\mathrm{p}}^{2} R_{\mathrm{p}}, \quad h_{\mathrm{p}}(\omega)=K_{\mathrm{p}}^{2} Z_{\mathrm{p}}(\omega)
$$

with the shunt impedance represented by the frequency dependent viscous coefficient $h_{\mathrm{p}}(\omega)$.

2.3. Resonant vibration absorber. The desired viscous function $h(\omega)$ in the common equivalent mechanical model must at least provide an inertance to create the desired resonant vibration absorber. In Fig. 3(a) the inertance is conveniently placed in series with the other components, whereby the shunt model precisely absorbs both intrinsic effects (Inoue et al., 2008) and the residual mode corrections considered in a following section. Thus, the apparent viscous function for the shunt impedance $h(\omega)=h_{\mathrm{s}}(\omega)$ is constructed by the reciprocal relation as

$$
\frac{1}{i \omega h_{\mathrm{s}}(\omega)}=\frac{1}{k_{\mathrm{s}}}+\frac{1}{i \omega c_{\mathrm{s}}}-\frac{1}{\omega^{2} m_{\mathrm{s}}}
$$

in which the two first terms represent supplemental shunt stiffness $k_{\mathrm{s}}$ and damping $c_{\mathrm{s}}$, introduced to alter the inherent transducer properties. When respectively introducing $h_{\mathrm{m}, \mathrm{p}}=h_{\mathrm{s}}$ in (6) and (9), the dynamic flexibility relation for the common vibration absorber can be written as

$$
x=\left(\frac{1}{k}+\frac{1}{i \omega c}-\frac{1}{\omega^{2} m}\right) f
$$

with Fig. 3(b) showing the resulting spring $(k)$, dashpot $(c)$ and inerter $(m)$ placed in the desired series arrangement.

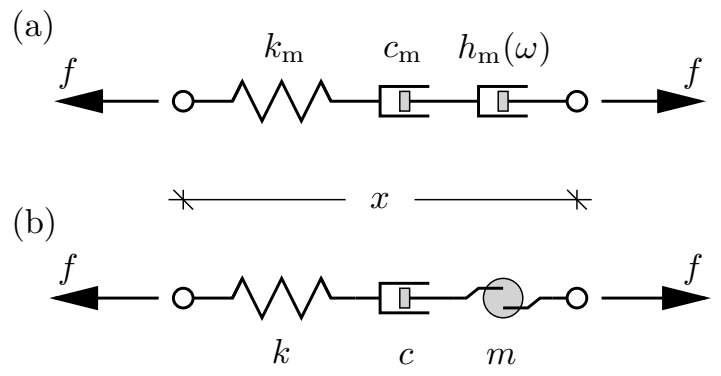

FIGURE 3. (a) Equivalent mechanical model of shunted electromagnetic transducer with spring $k_{\mathrm{m}}$, viscous damper $c_{\mathrm{m}}$ and frequency dependent dashpot $h_{\mathrm{m}}(\omega)$. (b) Desired absorber with spring $k$, dashpot $c$ and inerter $m$ in series. 


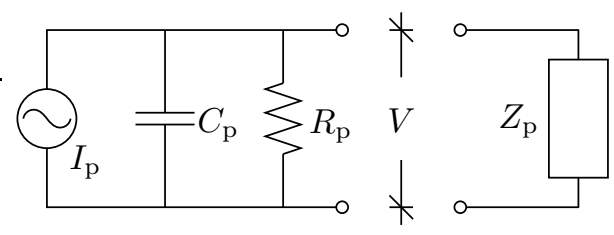

FiguRE 4. Circuit representation of piezoelectric transducer and shunt $Z_{\mathrm{p}}(\omega)$.
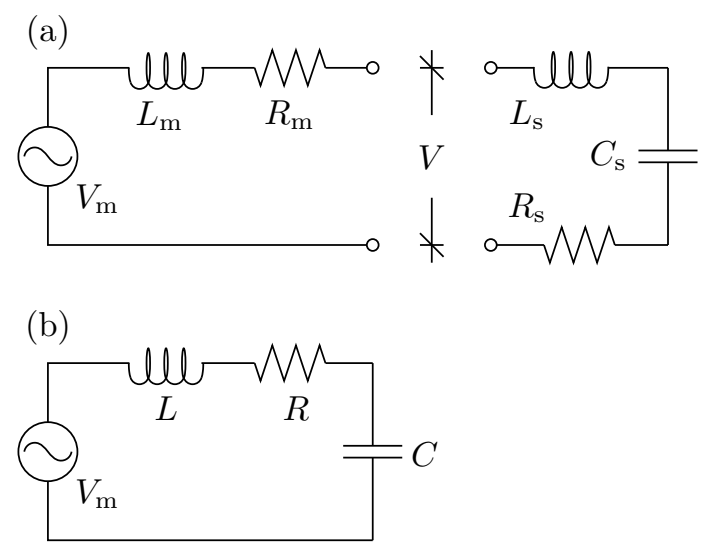

FiguRE 5. (a) Resonant electromagnetic shunt damper with (b) resulting electrical model.

2.3.1. Electromagnetic shunt. For the shunted electromagnetic transducer the desired vibration absorber relation (12) is constructed by $h_{\mathrm{m}}=h_{\mathrm{s}}$ in (11), which defines the series shunt in Fig. 5(a) with

$$
L_{\mathrm{s}}=\frac{K_{\mathrm{m}}^{2}}{k_{\mathrm{s}}}, \quad R_{\mathrm{s}}=\frac{K_{\mathrm{m}}^{2}}{c_{\mathrm{s}}}, \quad C_{\mathrm{s}}^{-1}=\frac{K_{\mathrm{m}}^{2}}{m_{\mathrm{s}}}
$$

introducing an equivalent shunt inductance, resistance and capacitance, respectively. Because the shunt in Fig. 5(a) is of series type, its components (with subscript s) are directly combined with the inherent transducer components (with subscript $\mathrm{m}$ ) to form the resulting model in Fig. 5(b) with

$$
L=L_{\mathrm{m}}+L_{\mathrm{s}}, R=R_{\mathrm{m}}+R_{\mathrm{s}}, C^{-1}=C_{\mathrm{s}}^{-1}
$$

defining the resulting inductance, resistance and capacitance, respectively. These resulting electrical components correspond to the mechanical components

$$
k=\frac{K_{\mathrm{m}}^{2}}{L}, \quad c=\frac{K_{\mathrm{m}}^{2}}{R}, \quad m=\frac{K_{\mathrm{m}}^{2}}{C^{-1}}
$$

of the desired vibration absorber model in Fig. 3(b). The analogy between shunt components and mechanical elements is summarized in Table 1. It follows from (14) and (15) that the absorber stiffness $k$ and damping $c$ are reduced by positive values of $L_{\mathrm{s}}$ and $R_{\mathrm{s}}$, whereby active shunts with negative inductance or resistance may be required to secure the desired absorber performance (Zhang et al., 2012; McDaid and Mace, 2013; Yan et al., 2016; Stabile et al., 2017). 
(a)

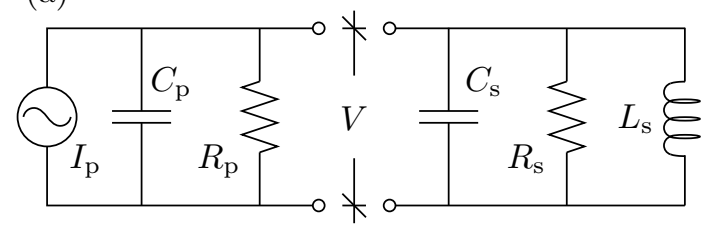

(b)

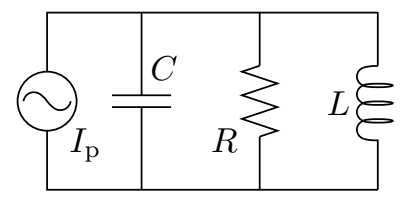

Figure 6. (a) Resonant piezoelectric shunt damper with (b) resulting electrical model.

2.3.2. Piezoelectric shunt. For resonant piezoelectric shunt damping the desired equivalence $h_{\mathrm{p}}=$ $h_{\mathrm{s}}$ in (11) determines the parallel shunt in Fig. 6(a) with shunt capacitance, resistance and inductance defined as

$$
C_{\mathrm{s}}^{-1}=\frac{k_{\mathrm{s}}}{K_{\mathrm{p}}^{2}}, \quad R_{\mathrm{s}}=\frac{c_{\mathrm{s}}}{K_{\mathrm{p}}^{2}}, \quad L_{\mathrm{s}}=\frac{m_{\mathrm{s}}}{K_{\mathrm{p}}^{2}}
$$

Because of the parallel shunt layout, the transducer and shunt components in Fig. 6(a) can be merged to form the resulting electrical model in Fig. 6(b) with

$$
\frac{1}{C^{-1}}=\frac{1}{C_{\mathrm{p}}^{-1}}+\frac{1}{C_{\mathrm{s}}^{-1}}, \frac{1}{R}=\frac{1}{R_{\mathrm{p}}}+\frac{1}{R_{\mathrm{s}}}, L=L_{\mathrm{s}}
$$

defining the mechanical absorber elements in Fig. 3(b) as

$$
k=K_{\mathrm{p}}^{2} C^{-1}, \quad c=K_{\mathrm{p}}^{2} R, \quad m=K_{\mathrm{p}}^{2} L
$$

which are also summarized in Table 1 . The transducer capacitance $C_{\mathrm{p}}$ is commonly so large for low-frequency structural vibrations, that the correspondingly large $L_{\mathrm{s}}$ must be realized by synthetic means (Fleming, 2004; Niederberger, 2005). Alternatively, a negative shunt capacitance $\left(C_{s}<0\right)$ may be added to reduce the inherent capacitance (de Marneffe and Preumont, 2008; Berardengo et al., 2016, b).

TABLE 1. Equivalence between vibration absorber and electromechanical shunt parameters.

\begin{tabular}{ccc}
\hline Absorber & Electromagnetic & Piezoelectric \\
\hline$k$ & $\frac{K_{\mathrm{m}}^{2}}{L_{\mathrm{m}}+L_{\mathrm{s}}}$ & $K_{\mathrm{p}}^{2} \frac{C_{\mathrm{p}}^{-1} C_{\mathrm{s}}^{-1}}{C_{\mathrm{p}}^{-1}+C_{\mathrm{s}}^{-1}}$ \\
$c$ & $\frac{K_{\mathrm{m}}^{2}}{R_{\mathrm{m}}+R_{\mathrm{s}}}$ & $K_{\mathrm{p}}^{2} \frac{R_{\mathrm{p}} R_{\mathrm{s}}}{R_{\mathrm{p}}+R_{\mathrm{s}}}$ \\
$m$ & $\frac{K_{\mathrm{m}}^{2}}{C_{\mathrm{s}}^{-1}}$ & $K_{\mathrm{p}}^{2} L_{\mathrm{s}}$ \\
\hline
\end{tabular}




\section{Structural Dynamics}

In a frequency domain representation, the discretized equations of motion for a flexible structure can be written as

$$
\left(-\omega^{2} \mathbf{M}+\mathbf{K}\right) \mathbf{u}+\mathbf{W} \mathbf{f}=\mathbf{f}_{e}
$$

in which $\mathbf{u}$ contains the complex amplitude of the structural degrees of freedom, $\mathbf{f}_{e}$ represents the external loading, while $\mathbf{M}$ and $\mathbf{K}$ are the mass and stiffness matrices. Structural damping is neglected in the present analysis. In (19) the vector $\mathbf{f}$ represents the transducer forces at individual locations determined by the participation array $\mathbf{W}$ with a column for each absorber. The collocated absorber displacements are therefore

$$
\mathbf{x}=\mathbf{W}^{T} \mathbf{u}
$$

with each component in $\mathbf{x}$ representing the displacement $x$ of an individual absorber. The composition of $\mathbf{W}$ depends on the transducer bonding to the structure, which in a finite element framework is described in detail for shunted piezoelectric transducers by e.g. Allik and Hughes (1970); Collet and Cunefare (2008); Thomas et al. (2009); Toftekær et al. (2019). For simplicity it is presently assumed that all absorbers are identical and governed by the common flexibility relation

$$
\mathbf{x}=\left(\frac{1}{k}+\frac{1}{i \omega c}-\frac{1}{\omega^{2} m}\right) \mathbf{f}
$$

similar to (12). The mechanical absorber parameters $k, c$ and $m$ are in the following calibrated to a specific vibration form, taking into account the interaction with non-resonant residual modes.

3.1. Eigenvalue problems. The vibration forms used in the subsequent modal reduction are governed by an underlying eigenvalue problem from an undamped form of the governing equations (19)-(21) without external load $\left(\mathbf{f}_{e}=\mathbf{0}\right)$.

3.1.1. Undamped vibration mode. In the limit without absorber damping $(c \rightarrow 0)$ the absorber force $\mathbf{f}=\mathbf{0}$, whereby (19) recovers the classic eigenvalue problem

$$
\left(-\omega_{j}^{2} \mathbf{M}+\mathbf{K}\right) \mathbf{u}_{j}=\mathbf{0} \quad, \quad \mathbf{f}=\mathbf{0}
$$

in which $\omega_{j}$ and $\mathbf{u}_{j}$ are respectively the natural frequency and mode shape vector of a vibration form $j$. For the electromagnetic transducer this eigenvalue problem corresponds to the open-circuit electrical limit without current $I=0$, obtained by $R_{\mathrm{S}} \rightarrow \infty$, while for the piezoelectric transducer it corresponds to shorted electrodes with $V=0$ given by $R_{\mathrm{S}} \rightarrow 0$. This eigenvalue problem (22) is in the following used to express the structural displacements and obtain the reduced single-mode model.

3.1.2. Structure-absorber mode. In the opposite limit $c \rightarrow \infty$ the dashpot in Fig. 3(b) becomes rigid and instantaneously transfers the absorber force in $\mathbf{f}$, whereby (21) governs a second order 
dynamic equation that requires additional dofs $(\mathbf{f} / k)$ in the augmented eigenvalue problem

$$
\left(\left[\begin{array}{cc}
\mathbf{K} & k \mathbf{W} \\
\mathbf{0}^{T} & k \mathbf{I}
\end{array}\right]-\tilde{\omega}_{j}^{2}\left[\begin{array}{cc}
\mathbf{M} & \mathbf{0} \\
-m \mathbf{W}^{T} & m \mathbf{I}
\end{array}\right]\right)\left[\begin{array}{c}
\mathbf{u} \\
\mathbf{f} / k
\end{array}\right]_{j}=\left[\begin{array}{l}
\mathbf{0} \\
\mathbf{0}
\end{array}\right]
$$

In practice with finite values of $R_{\mathrm{m}}$ or $R_{\mathrm{p}}$ the limit $c \rightarrow \infty$ is not strictly available by either $R_{\mathrm{s}} \rightarrow 0$ for the electromagnetic shunt or $R_{\mathrm{S}} \rightarrow \infty$ for the piezoelectric shunt. However, for proper values of the absorber stiffness $k$ and inertance $m$ this augmented eigenvalue problem can determine two natural frequencies $\tilde{\omega}_{j}$ for the targeted vibration mode $j=r$ that by frequency matching will be used in the following to consistently calibrate the supplemental residual mode correction terms.

3.1.3. Locked vibration mode. In the above limit $(c \rightarrow \infty)$ the augmented eigenvalue problem (23) recovers the classic problem in (22) for $m \rightarrow 0$, while for $m \rightarrow \infty$ it instead includes the absorber stiffness $k$,

$$
\left(-\hat{\omega}_{j}^{2} \mathbf{M}+\mathbf{K}+k \mathbf{W} \mathbf{W}^{T}\right) \hat{\mathbf{u}}_{j}=\mathbf{0} \quad, \quad \mathbf{f}=k \mathbf{W}^{T} \hat{\mathbf{u}}_{j}
$$

so that $\hat{\omega}_{j} \geq \omega_{j}$. For the electromagnetic transducer this eigenvalue problem corresponds to shortcircuited electrodes with $V=0$, whereas in the piezoelectric case it corresponds to open-circuit conditions enforced by $I=0$. For a reduced single-mode model, this idealized eigenvalue problem is commonly used to identify an effective or modal electromechanical coupling coefficient, although the inherent resistance $\left(R_{\mathrm{m}}\right.$ or $\left.R_{\mathrm{p}}\right)$ must be neglected to fully recover $(24)$.

3.2. Modal representation. The structural vibration amplitude $\mathbf{u}$ is now represented by the linear combination

$$
\mathbf{u}=\sum_{j} \frac{\mathbf{u}_{j}}{\nu_{j}} u_{j}, \quad \nu_{j}^{2}=\mathbf{u}_{j}^{T} \mathbf{W} \mathbf{W}^{T} \mathbf{u}_{j}
$$

of the undamped mode shapes $\mathbf{u}_{j}$ from (22), conveniently normalized by the (square-root of the) modal strain energy factor $\nu_{j}^{2}$. The modal representation (25) is substituted into (19), which is then pre-multiplied with $\mathbf{u}_{r}^{T} / \nu_{r}$ to provide the modal equation of motion

$$
\left(-\omega^{2} m_{r}+k_{r}\right) u_{r}+f_{r}=f_{r}^{e}
$$

in which modal mass, stiffness and load are defined as

$$
m_{r}=\frac{1}{\nu_{r}^{2}} \mathbf{u}_{r}^{T} \mathbf{M} \mathbf{u}_{r}, \quad k_{r}=\frac{1}{\nu_{r}^{2}} \mathbf{u}_{r}^{T} \mathbf{K} \mathbf{u}_{r}, \quad f_{r}^{e}=\frac{1}{\nu_{r}} \mathbf{u}_{r}^{T} \mathbf{f}_{\mathrm{e}}
$$

while

$$
f_{r}=\frac{1}{\nu_{r}} \mathbf{u}_{r}^{T} \mathbf{W} \mathbf{f}
$$

is the modal absorber force.

3.3. Effective electromechanical coupling coefficient. An effective or modal Electro-Mechanical Coupling Coefficient (EMCC) $K_{r}$ is determined by combining the classic eigenvalue problem in (22) with the locked eigenvalue problem (24) that includes the absorber stiffness $k$. When the latter is pre-multiplied by $\mathbf{u}_{r}^{T}$, it can be written as

$$
-\hat{\omega}_{r}^{2} \mathbf{u}_{r}^{T} \mathbf{M} \hat{\mathbf{u}}_{r}+\mathbf{u}_{r}^{T} \mathbf{K} \hat{\mathbf{u}}_{r}+k \mathbf{u}_{r}^{T} \mathbf{W} \mathbf{W}^{T} \hat{\mathbf{u}}_{r}=\mathbf{0}
$$


The classic eigenvalue problem (22) is then pre-multiplied with $\hat{\mathbf{u}}_{j}^{T}$, whereby it can be used to eliminate the second term in (29) with the stiffness matrix. This combined equation can then be used to express the effective EMCC as (Toftekær et al., 2019)

$$
K_{r}^{2}=\frac{\hat{\omega}_{r}^{2}-\omega_{r}^{2}}{\omega_{r}^{2}}=\frac{k}{k_{r}} \frac{m_{r} \mathbf{u}_{r}^{T} \mathbf{W} \mathbf{W}^{T} \hat{\mathbf{u}}_{r}}{\mathbf{u}_{r}^{T} \mathbf{M} \hat{\mathbf{u}}_{r}}
$$

For a pure single-mode structure without modal spill-over from other modes, the last fraction becomes unity and $K_{r}^{2}=k / k_{r}$. However, for flexible structures with residual vibration modes influencing the absorber characteristics, the second fraction in (30) describes the modal interaction that next will be represented by two supplemental modal correction terms.

\section{Residual Mode CORRECTION}

The absorber displacements $\mathbf{x}=\mathbf{W}^{T} \mathbf{u}$ in (21) is commonly approximated by the single-mode displacement $\mathbf{x}_{r}=\nu_{r}^{-1} \mathbf{W}^{T} \mathbf{u}_{r} u_{r}$, although the influence from residual modes can be accurately represented by an augmented representation (Krenk and Høgsberg, 2016),

$$
\mathbf{x}_{r}=\mathbf{x}+\left(\frac{1}{\kappa_{r}^{\prime} k_{r}}-\frac{1}{\mu_{r}^{\prime} m_{r} \omega^{2}}\right) \mathbf{f}
$$

with additional modal flexibility and inertia governed by the coefficients $\kappa_{r}^{\prime}$ and $\mu_{r}^{\prime}$, respectively. First the modal displacement $\mathbf{x}_{r}=\nu_{r}^{-1} \mathbf{W}^{T} \mathbf{u}_{r} u_{r}$ is eliminated in (31), whereafter $\mathbf{x}$ is substituted from (21). When pre-multiplied by $\nu_{r}^{-1} \mathbf{u}_{r}^{T} \mathbf{W}$ the relation can then be written as

$$
u_{r}=\left(\frac{1}{\kappa_{r} k_{r}}+\frac{1}{i \omega \beta_{r} \sqrt{k_{r} m_{r}}}-\frac{1}{\mu_{r} m_{r} \omega^{2}}\right) f_{r}
$$

with $f_{r}$ defined in (28) and the resulting modal stiffness, mass and damper ratios introduced as

$$
\frac{1}{\kappa_{r}}=\frac{k_{r}}{k}+\frac{1}{\kappa_{r}^{\prime}} \quad, \quad \frac{1}{\mu_{r}}=\frac{m_{r}}{m}+\frac{1}{\mu_{r}^{\prime}} \quad, \quad \beta_{r}=\frac{c}{\sqrt{k_{r} m_{r}}}
$$

The modal flexibility relation (32) constitutes the modal absorber model in Fig. 7, with the modal stiffness $\kappa_{r} k_{r}$, damping $\beta_{r} \sqrt{k_{r} m_{r}}$ and inertance $\mu_{r} m_{r}$ explicitly included by the series and parallel shunts applied in Figs. 5(a) and 6(a), respectively.

4.1. Single-mode calibration. The single-mode equations (26) and (32) can be combined by eliminating $f_{r}$, which yields the frequency response function

$$
\frac{u_{r} k_{r}}{f_{r}^{e}}=\frac{-\xi^{2} \mu_{r}+i \xi \frac{\kappa_{r} \mu_{r}}{\beta_{r}}+\kappa_{r}}{\left(-\xi^{2}+1\right)\left(-\xi^{2} \mu_{r}+i \xi \frac{\kappa_{r} \mu_{r}}{\beta_{r}}+\kappa_{r}\right)-\xi^{2} \kappa_{r} \mu_{r}}
$$

FiguRE 7. Resonant vibration absorber with modal parameters and modal displacement $u_{r}$. 
with the non-dimensional frequency ratio

$$
\xi=\frac{\omega}{\omega_{r}}=\omega \sqrt{\frac{m_{r}}{k_{r}}}
$$

Several calibration principles are available for an apparent single-mode structure, with a few summarized in Table 2 for a prescribed stiffness ratio $\kappa_{r}$. The specific transducer and shunt components can subsequently be determined by the equivalence relations in Table 1.

The free vibration properties of the single-mode system are represented by the complex root $\omega$ of the characteristic equation, obtained from the denominator in (34). Figure 8(a) shows the complex root trajectories (loci) with $\beta$ as gain and $\mu_{r}$ determined by the four calibration principles in Table 2. Equal modal damping is associated with a bifurcation point and obtained by $\mu_{r}=\kappa_{r}$ in Table 2 , for which 'Maximum damping' chooses $\beta_{r}$ exactly at the bifurcation point (red o), while 'Balanced calibration' (blue $*$ ) suggests a slightly reduced attainable damping by a larger $\beta_{r}$ to avoid the modal interference at bifurcation. For the corresponding dynamic amplification curves in Fig. 8(b) the 'Minimum amplitude' (black) and 'Balanced calibration' (blue) secure almost flat plateaus, whereby the 'Balanced calibration' principle is chosen because it combines equal damping and a flat amplitude response curve.

4.2. Correction ratios by frequency matching. The residual mode correction coefficients $\kappa_{r}^{\prime}$ and $\mu_{r}^{\prime}$ in (31) are determined by a novel frequency matching principle with the modal frequencies of the full flexible structure, obtained numerically from the eigenvalue problem (23) or experimentally by peaks in a measured response spectrum.

4.2.1. Structure-absorber mode. The free vibration characteristics of the structure with rigid absorber dashpots $(c \rightarrow \infty)$ is governed by the eigenvalue problem (23). For $N_{a}$ absorbers calibrated to the target mode $j=r$, the eigenvalue problem (23) determines $N_{a}+1$ natural frequencies associated with the targeted vibration form. Two of these are associated with coupled absorber-structure vibrations, while the remaining $N_{a}-1$ represent pure absorber modes that are highly damped and with limited structural interaction. Among the $N_{a}+1$ roots, the two dynamic frequency ratios $\xi_{A}$

TABLE 2. Calibration formulae for vibration absorber.

\begin{tabular}{lcc}
\hline Principle & $\mu_{r}$ & $\beta_{r}$ \\
\hline Minimum amplitude (Wu, 1996) & $\frac{\kappa_{r}}{1-\frac{1}{2} \kappa_{r}}$ & $\sqrt{\frac{1}{2} \kappa_{r}}$ \\
Fixed point (Yamada et al., 2010) & $\frac{\kappa_{r}}{1-\frac{1}{2} \kappa_{r}}$ & $\sqrt{\frac{2}{3} \kappa_{r}}$ \\
Maximum damping (Caruso, 2001) & $\kappa_{r}$ & $\sqrt{\frac{1}{4} \kappa_{r}}$ \\
Balanced calibration (Høgsberg and Krenk, 2012) & $\kappa_{r}$ & $\sqrt{\frac{1}{2} \kappa_{r}}$ \\
\hline
\end{tabular}



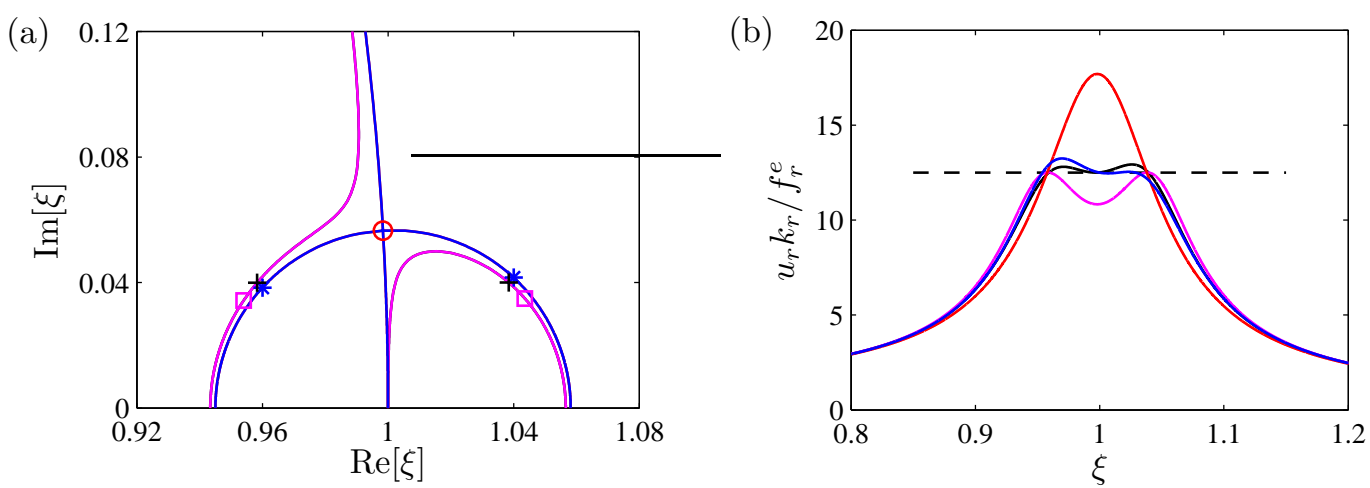

FiguRE 8. (a) Complex root trajectories and dynamic amplification curves (b) for a single-mode system obtained by the calibration principles in Table 2: Minimum amplitude (black, + ), fixed point (magenta, $\square$ ), maximum damping (red,o) and balanced calibration (blue,*).

and $\xi_{B}$ are commonly identified as the smallest and largest, while the intermediate roots represent absorber modes.

The characteristic equation is obtained from the denominator of the modal frequency response function (34). For $\beta_{r} \rightarrow \infty$ it reduces to the quadratic polynomial

$$
\xi^{4}-\left(1+\tilde{\kappa}_{r}+\frac{\tilde{\kappa}_{r}}{\tilde{\mu}_{r}}\right) \xi^{2}+\frac{\tilde{\kappa}_{r}}{\tilde{\mu}_{r}}=0
$$

with roots $\xi^{2}=\xi_{A}^{2}$ and $\xi_{B}^{2}$ obtained from (23) with appropriate estimates of the absorber stiffness $k=\tilde{k}$ and inertance $m=\tilde{m}$. The sum and product of the two roots can be written as

$$
\xi_{A}^{2}+\xi_{B}^{2}=1+\tilde{\kappa}_{r}+\frac{\tilde{\kappa}_{r}}{\tilde{\mu}_{r}} \quad, \quad \xi_{A}^{2} \xi_{B}^{2}=\frac{\tilde{\kappa}_{r}}{\tilde{\mu}_{r}}
$$

whereby the corresponding absorber ratios can be determined as

$$
\tilde{\kappa}_{r}=\left(1-\xi_{A}^{2}\right)\left(\xi_{B}^{2}-1\right) \quad, \quad \tilde{\mu}_{r}=\left(1-\xi_{B}^{-2}\right)\left(\xi_{A}^{-2}-1\right)
$$

From (33) the correction ratios are then explicitly obtained as

$$
\frac{1}{\kappa_{r}^{\prime}}=\frac{1}{\tilde{\kappa}_{r}}-\frac{k_{r}}{\tilde{k}} \quad, \quad \frac{1}{\mu_{r}^{\prime}}=\frac{1}{\tilde{\mu}_{r}}-\frac{m_{r}}{\tilde{m}}
$$

for assumed absorber parameters $\tilde{k}$ and $\tilde{m}$, and with $\tilde{\kappa}_{r}$ and $\tilde{\mu}_{r}$ obtained by frequency matching from (38). The accuracy of these expressions depends on how well the estimated absorber components $\tilde{k}$ and $\tilde{m}$ coincide with $k$ and $m$ in the final absorber design. Thus, the proposed frequency matching procedure may be used iteratively to improve the accuracy. A similar identification of the electromechanical coupling from the two resonances associated with piezoelectric $L$ shunts has previously been proposed by Porfiri et al. (2007).

4.2.2. Locked absorber mode. The locked eigenvalue problem in (24) corresponds to $c \rightarrow \infty$ and $m \rightarrow \infty$ and thus $\beta_{r} \rightarrow \infty$ and $\mu_{r}=\mu_{r}^{\prime}$. In this particular limit the characteristic equation recovers (36) with $\tilde{\mu}_{r}=\mu_{r}^{\prime}$. Thus, the modal equation remains a quadratic polynomial in $\xi^{2}$, although (24) only defines a single root $\xi^{2}=\hat{\xi}^{2}$. However, for absorber configurations with limited residual mode inertia $\left(\mu_{r}^{\prime} \rightarrow \infty\right)$ the ratio $\tilde{\kappa}_{r} / \mu_{r}^{\prime} \rightarrow 0$, whereby the roots to the characteristic equation 
become $\hat{\xi}_{r}^{2}=0$ and $1+\tilde{\kappa}_{r}$. The latter non-trivial solution then determines the modal EMCC in $(30)$ as

$$
K_{r}^{2}=\hat{\xi}_{r}^{2}-1=\tilde{\kappa}_{r}
$$

whereby the quasi-static residual mode coefficients can be obtained from (39) as

$$
\frac{1}{\kappa_{r}^{\prime}}=\frac{1}{K_{r}^{2}}-\frac{k_{r}}{\tilde{k}} \quad, \quad \frac{1}{\mu_{r}^{\prime}}=0
$$

This quasi-static residual mode correction has been found to provide very accurate calibration results for laminate-type piezoelectric transducers on flexible beams and plates (Toftekær et al., 2019; Toftekær and Høgsberg, 2020).

4.3. Design procedure. A design is now proposed for the 'balanced calibration' in Table 2, by which the stiffness ratio

$$
\kappa_{r}=8 \zeta_{\mathrm{des}}^{2}
$$

is initially obtained from a desired damping ratio $\zeta_{\text {des }}$ (Krenk and Høgsberg, 2013), while

$$
\mu_{r}=\kappa_{r} \quad, \quad \beta_{r}=\sqrt{\frac{1}{2} \kappa_{r}}
$$

follow directly from Table 2 .

The classic eigenvalue problem (22) is then solved and the absorber stiffness and inertance are estimated as

$$
\tilde{k}=\kappa_{r} k_{r} \quad, \quad \tilde{m}=\mu_{r} m_{r}
$$

thus initially neglecting any influence from residual modes. For finite values of $\mu_{r}^{\prime}$ the frequency ratios $\xi_{A}$ and $\xi_{B}$ are obtained by solving the augmented eigenvalue problem (23), whereafter $\kappa_{r}^{\prime}$ and $\mu_{r}^{\prime}$ are determined by (38) and (39). For the quasi-static representation, the locked eigenvalue problem (24) instead determines the (squared) modal EMCC $K_{r}^{2}$ by (30), which subsequently determines $\kappa_{r}^{\prime}$ and $\mu_{r}^{\prime}$ by (41). The actual absorber parameters $k, m$ and $c$ are then determined by the three expressions in (33), respectively, while the actual shunt inductance $L_{\mathrm{s}}$, resistance $R_{\mathrm{s}}$ and capacitance $C_{\mathrm{s}}$ are finally obtained from Table 1.

\section{DAMPING OF A FLOATING TRUSS}

The geometry of a 3D truss structure is defined in Fig. 9 and inspired by a NASA research model (McGowan et al., 1990) with transverse dimensions $a$ and length $10 a$, axial stiffness $E A$ and mass per unit length $\rho A$ for each bar. The truss consists of 135 bars that connect 44 nodes, whereby the model contains $N_{\text {dof }}=132$ degrees-of-freedom (dofs).

Piezoelectric stack actuators are inserted as load carrying members in the blue, green and magenta bar elements in Fig. 9. For these compound elements the element length $\ell=\ell_{\mathrm{b}}+\ell_{\mathrm{t}}$ is the sum of the individual lengths $\ell_{\mathrm{b}}$ and $\ell_{\mathrm{t}}$ of respectively the bar (b) and transducer (t) parts. The resulting stiffness and mass of the six compound element are for simplicity assumed to equal $E A$ and $\rho A$, respectively, whereby all 135 bars in the truss are mechanically identical and only differ in length. 


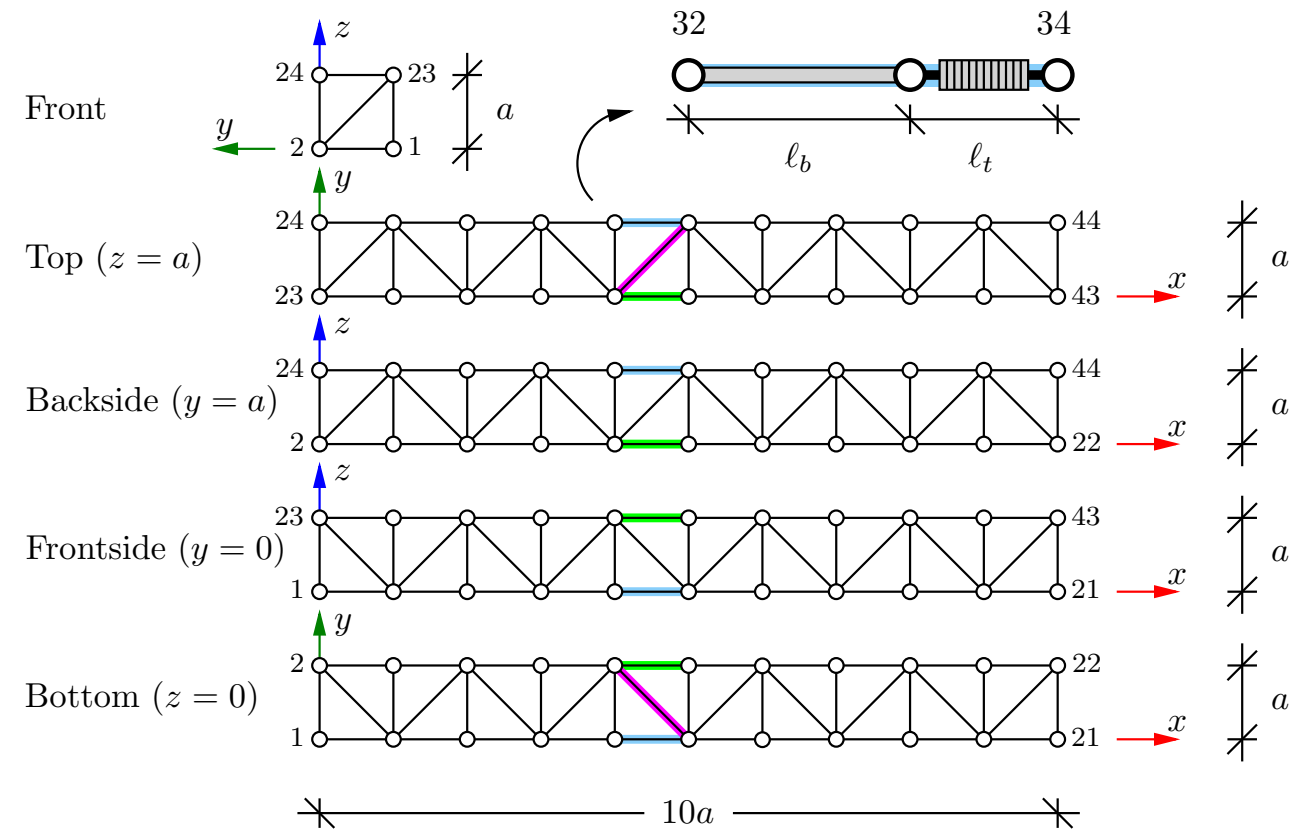

Figure 9. Architecture of generic 10-bay truss with absorbers elements identified by blue, green (bending) and magenta (torsion) bars.

The truss is unsupported with six rigid body modes, and index $j=1,2, \ldots$ only refers to the vibratory modes with non-vanishing frequencies. Figure 10 shows the first three vibration forms ( $j=1$ to 3 ), identified as two bending modes $(j=1$ and 2$)$ and a torsional mode $(j=3)$. The natural frequencies are $\omega_{j} / \omega_{\mathrm{t}}=0.0147,0.0148$ and 0.0208 , relative to $\omega_{\mathrm{t}}=(\pi / \ell) \sqrt{E / \rho}$ for a single bar. The small difference in the two bending frequencies occurs because of the diagonals in the cross-sectional $\{y, z\}$-planes.

The three pairs of compound bars are installed to damp the first three vibrations modes, respectively. In Fig. 9 the two blue and the two green bars target respectively the first and the second bending mode ( $r=1$ and 2), while the remaining two magenta (diagonal) bars damp the first torsional mode $(r=3)$. The three participation arrays $\mathbf{W}_{r}$ for $r=1$ to 3 have dimensions $N_{\text {dof }} \times 2$ and are associated with the three $2 \times 1$ force vectors $\mathbf{f}_{r}$. The entries in $\mathbf{W}_{1}$ and $\mathbf{W}_{2}$ for the horizontal (blue and green) bars are \pm 1 , while in $\mathbf{W}_{3}$ for the magenta bars the entries are reduced to $\pm \frac{1}{2} \sqrt{2}$ to compensate for the longer diagonal length.

The particular absorber locations are chosen to reduce the modal coupling between the three targeted vibration modes. Table 3 shows the modal mass ratio $m_{s} / m_{j}^{*}$ for each vector array $\mathbf{W}=\mathbf{W}_{r}$, with $m_{s}$ representing the total translational mass of the unsupported truss. The diagonal structure of the table entries indicate perfect isolation of the two bending modes, while a small modal coupling is observed for the diagonal (magenta) absorber bars represented by $\mathbf{W}_{3}$. The calibration is based on a desired damping ratio $\zeta_{\text {des }}=0.05$, whereby $\mu_{r}=\kappa_{r}=0.0200$ and $\beta_{r}=0.1000$ follow from (42) and (43) for all absorbers. The absorber parameters for the 


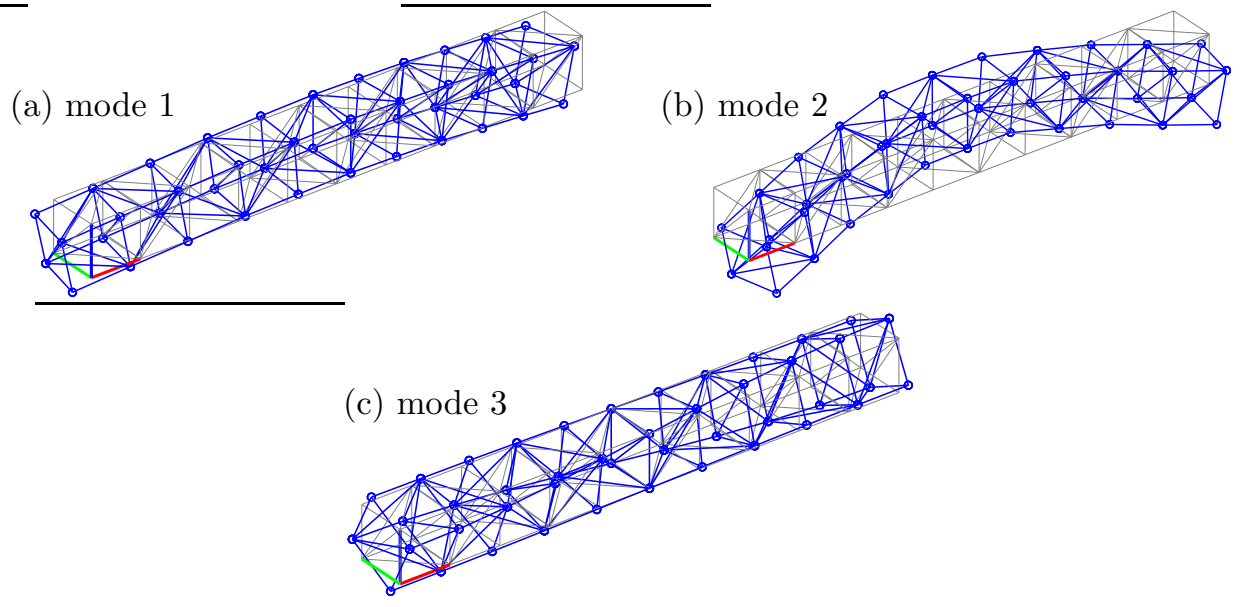

FigURE 10. First three vibration modes of generic truss without supports.

three calibrations $(r=1$ to 3$)$ are presented in Table 4. It is seen that for all three modes the inertia correction coefficient $\mu_{r}^{\prime}$ is much larger than $\mu_{r}=0.0200$, whereby the actual absorber mass ratio $m / m_{r} \simeq \mu_{r}$. However, the stiffness correction represented by $\kappa_{r}^{\prime}$ appears to have a significant impact on the calibration, in particular for the torsional mode $(r=3)$ with $\kappa_{3}^{\prime}<\kappa_{3}$ and the actual absorber stiffness ratio $k / k_{3}$ being almost $21 \%$ larger than $\kappa_{3}=0.0200$. Table 4 also provides the calibration results for the quasi-static residual mode correction with assumed $\mu_{r}^{\prime} \rightarrow \infty$ and only a marginal reduction in absorber stiffness. For optimal values of $k / k_{r}$ and $m / m_{r}$ from Table 4 , the (piezoelectric) shunt capacitance and inductance are determined from the last column of Table 1 as

$$
\frac{C_{\mathrm{p}}}{C_{\mathrm{s}}}=\frac{k / k_{r}}{K_{\mathrm{p}}^{2} /\left(k_{r} C_{\mathrm{p}}\right)-k / k_{r}} \quad, \quad L_{\mathrm{s}} C_{\mathrm{p}} \omega_{r}=\frac{m / m_{r}}{K_{\mathrm{p}}^{2} /\left(k_{r} C_{\mathrm{p}}\right)}
$$

while $\beta_{r}$ correspondingly determines the shunt resistance

$$
R_{\mathrm{s}} C_{\mathrm{p}} \omega_{r}=\frac{\beta_{r}}{K_{\mathrm{p}}^{2}\left(k_{r} C_{\mathrm{p}}\right)^{-1}-\beta_{r} /\left(R_{\mathrm{p}} C_{\mathrm{p}} \omega_{r}\right)}
$$

Note that negative values of $C_{\mathrm{s}}$ and $R_{\mathrm{S}}$ must be produced actively in the shunt when the respective denominators in (45) and (45) become negative.

The complex roots for the full flexible structure are obtained by solving the eigenvalue problem associated with the homogeneous form of the governing equations (19) to (21). The magnitude of the normalized natural frequency $\left|\xi_{j}\right|$ and the associated damping ratio $\zeta_{j}=\operatorname{Im}\left[\xi_{j}\right] /\left|\xi_{j}\right|$ are presented in Table 5 for the three targeted modes $r=1$ to 3 . The table is divided into three parts (top, middle and bottom). In the top part the mode $r$ is only damped by $\mathbf{W}=\mathbf{W}_{r}$.

TABLE 3 . Modal mass $m_{s} / m_{r}$ ratio for connectivity arrays $\mathbf{W}=\mathbf{W}_{r}$.

\begin{tabular}{cccc}
\hline$j$ & $r=1$ & $r=2$ & $r=3$ \\
\hline 1 & 0.0709 & 0.0000 & 0.0004 \\
2 & 0.0000 & 0.0615 & 0.0003 \\
3 & 0.0000 & 0.0000 & 0.0885 \\
\hline
\end{tabular}


Because only two absorbers act simultaneously for each $r$, the top part presents the first five modes: Two vibration modes for the targeted vibration form (A and B), a single absorber mode (C) and two (undamped) modes $(j \neq r)$ not targeted by the absorber configuration in $\mathbf{W}_{r}$. The individual absorber pairs provide the desired damping ratio $\left(\zeta_{r}=0.050\right)$ to the targeted modes (A) and (B), while the damping ratio of the absorber mode (C) is approximately twice the desired value. Furthermore, the non-targeted modes are not influenced by the individual absorber pairs, indicating that the individual absorber calibration might also be accurate in the combined case with all three absorber pairs installed simultaneously in the truss. Because of the limited influence from the residual mode inertia, the quasi-static calibration (in parentheses) results in only slightly reduced damping ratios.

The three absorber pairs are now installed simultaneously in the truss, implemented by the full $N_{\text {dof }} \times 6$ participation array $\mathbf{W}=\left[\mathbf{W}_{1} \mathbf{W}_{2} \mathbf{W}_{3}\right]$. In the middle part of Table 5 the natural frequencies and damping ratios are therefore determined for the first nine modes: Six targeted vibration modes and three absorber modes. The nine modes are classified with respect to $r$ by inspection of the associated vibration forms. It is seen that for all three modes the desired damping ratio $\zeta_{\text {des }}=0.050$ is practically reproduced for the vibrational modes, while the three absorber modes have damping ratios that are approximately twice the desired damping level. Thus, the proposed individual absorber calibration is effectively carried on to simultaneous damping of the three lowest vibration modes.

As demonstrated by Thomas et al. (2012) the performance or attainable damping of piezoelectric shunt damping is determined by the modal electromechanical coupling coefficient $\kappa_{r}$ when neglecting the inertia correction, as in (40) - (41). However, the inclusion of residual coefficients $\kappa_{r}^{\prime}$ and $\mu_{r}^{\prime}$ imply that the actual absorber ratios $k / k_{r}$ and $m / m_{r}$ will deviate from $\kappa_{r}$ and $\mu_{r}$ so that the desired damping $\zeta_{\text {des }}$ in (42) is retained. The influence of the residual mode coefficients are investigated in the bottom part of Table 5 for the simultaneous damping case with absorber stiffness $k=\kappa_{r} k_{r}$ and inertance $m=\mu_{r} m_{r}$ neglecting the residual mode corrections. It is seen that for $r=1$ to 3 , the attainable damping ratios $\zeta_{5}=0.0324, \zeta_{6}=0.0293$ and $\zeta_{3}=0.0200$ are substantially reduced compared to $\zeta_{\text {des }}=0.0500$, demonstrating the sensitivity with respect to the non-resonant modes of the flexible structure.

\section{Conclusion}

The mechanical equivalence between parallel $R L$ shunt damping with piezoelectric transducers and series $R C$ shunt damping by electromagnetic transducers is used to construct a common vibration absorber format with a spring, dashpot and inerter placed in series. The equivalence implies that tuning formulae for piezoelectric parallel $R L$ shunts can be directly applied for electromagnetic series $R C$ shunts, and vice versa. The theory is formulated for multiple absorbers targeting a specific mode of a flexible structure, for which the interaction with residual modes is represented by two supplemental modal displacements. The particular shunt configurations are specifically chosen because they consistently incorporate both intrinsic shunt properties and the residual mode 
TABLE 4. Natural frequency ratios and absorber parameters for $\mu_{r}=\kappa_{r}=0.0200$.

\begin{tabular}{cccc}
\hline & $r=1$ & $r=2$ & $r=3$ \\
\hline$\xi_{A}$ & 0.9141 & 0.9097 & 0.8900 \\
$\xi_{C}$ & 0.9636 & 0.9589 & 0.9033 \\
$\xi_{B}$ & 1.0550 & 1.0516 & 1.0402 \\
$\kappa_{r}^{\prime}$ & 0.2613 & 0.2089 & 0.1153 \\
$\mu_{r}^{\prime}$ & 19.210 & 7.4180 & 3.4204 \\
$k / k_{r}$ & 0.0217 & 0.0221 & 0.0242 \\
$m / m_{r}$ & 0.0200 & 0.0201 & 0.0201 \\
\hline$\hat{\xi}$ & 1.0093 & 1.0091 & 1.0085 \\
$K_{r}^{2}$ & 0.0186 & 0.0183 & 0.0171 \\
$\kappa_{r}^{\prime}$ & 0.2649 & 0.2150 & 0.1193 \\
$\mu_{r}^{\prime}$ & $\infty$ & $\infty$ & $\infty$ \\
$k / k_{r}$ & 0.0216 & 0.0221 & 0.0240 \\
$m / m_{r}$ & 0.0200 & 0.0200 & 0.0200 \\
\hline
\end{tabular}

TABLE 5. Damping ratio and natural frequency from individual absorber pairs (top), combined absorbers (middel) and combined absorbers without residual mode correction (bottom), with $\mu_{r}=\kappa_{r}=0.0200$ and $\beta_{r}=0.1000$.

\begin{tabular}{|c|c|c|c|c|c|c|}
\hline$j$ & $\left|\xi_{j}\right|$ & $\begin{array}{c}r=1 \\
\zeta_{j}\end{array}$ & $\left|\xi_{j}\right|$ & $\begin{array}{c}r=2 \\
\zeta_{j} \\
\end{array}$ & $\left|\xi_{j}\right|$ & $\begin{array}{l}r=3 \\
\zeta_{j}\end{array}$ \\
\hline 1 & $0.9512(\mathrm{~A})$ & $0.0499(0.0499)$ & $0.9512(\mathrm{~A})$ & $0.0499(0.0498)$ & 0.7089 & $0.0000(0.0000)$ \\
\hline 2 & $0.9993(\mathrm{C})$ & $0.0999(0.0998)$ & 0.9932 & $0.0000(0.0000)$ & 0.7138 & $0.0000(0.0000)$ \\
\hline 3 & 1.0068 & $0.0000(0.0000)$ & $1.0028(\mathrm{C})$ & $0.1004(0.1002)$ & $0.9513(\mathrm{~A})$ & $0.0499(0.0496)$ \\
\hline 4 & $1.0513(\mathrm{~B})$ & $0.0499(0.0499)$ & $1.0514(\mathrm{~B})$ & $0.0499(0.0498)$ & $0.9712(\mathrm{C})$ & $0.0956(0.0951)$ \\
\hline 5 & 1.4105 & $0.0000(0.0000)$ & 1.4009 & $0.0000(0.0000)$ & $1.0514(\mathrm{~B})$ & $0.0499(0.0496)$ \\
\hline 1 & 0.9512 & $0.0499(0.0498)$ & & & & \\
\hline 2 & & & 0.9512 & $0.0499(0.0498)$ & & \\
\hline 3 & 0.9967 & $0.0995(0.0994)$ & & & & \\
\hline 4 & & & 1.0052 & $0.1008(0.1006)$ & & \\
\hline 5 & 1.0513 & $0.0500(0.0500)$ & & & & \\
\hline 6 & & & 1.0513 & $0.0500(0.0498)$ & & \\
\hline 7 & & & & & 0.9515 & $0.0498(0.0495)$ \\
\hline 8 & & & & & 0.9713 & $0.0956(0.0951)$ \\
\hline 9 & & & & & 1.0515 & $0.0501(0.0498)$ \\
\hline 1 & 0.9290 & 0.0638 & & & & \\
\hline 2 & & & 0.9233 & 0.0660 & & \\
\hline 3 & 0.9595 & 0.0955 & & & & \\
\hline 4 & & & 0.9630 & 0.0966 & & \\
\hline 5 & 1.0379 & 0.0324 & & & & \\
\hline 6 & & & 1.0360 & 0.0293 & & \\
\hline 7 & & & & & 0.8991 & 0.0720 \\
\hline 8 & & & & & 0.9036 & 0.0890 \\
\hline 9 & & & & & 1.0301 & 0.0200 \\
\hline
\end{tabular}

corrections, while the opposite shunts will not. The two residual mode coefficients are determined by a new frequency matching principle based on frequencies for the structure-absorber modes for the system with rigid absorber dashpots. For piezoelectric shunt damping these limiting frequencies are associated with infinite (shunt) resistance, while in the case of electromagnetic shunt 
damping they are determined for vanishing resistance. An alternative quasi-static residual mode correction is determined for rigid absorber dashpot and inerter elements, whereby the flexibility correction can be directly related to the change in the modal stiffness ratio relative to the modal electromechanical coupling coefficient (EMCC). The accuracy and robustness of the shunt calibration procedure is numerically verified for an unsupported truss structure, for which the residual mode correction recovers the desired damping ratio, even though the structure possesses six rigid body modes.

\section{FUNDING}

The author acknowledges the financial support of The Danish Council for Independent Research (DFF) via the FP1 grant 6111-00132A: Resonant Piezoelectric Shunt Damping of Structures.

\section{Declaration of Conflicting Interests}

The author declares that there is no conflict of interest.

\section{REFERENCES}

Allik H and Hughes TJR (1970) Finite element method for piezoelectric vibration. International Journal for Numerical Methods in Engineering 2: 151-157.

Behrens S, Fleming AJ and Moheimani SOR (2003) Electromagnetic shunt damping. Proceddings of the 2003 IEEE/ASME International Conference on Advanced Intelligent Mechatronics (AIM 2003), Kobe, Japan: 1145-1150.

Behrens S, Fleming AJ and Moheimani SOR (2005) Passive vibration control via electromagnetic shunt damping. IEEE/ASME Transactions on Mechatronics 10: 118-122.

Berardengo M, Manzoni S and Vanali M (2016) The behaviour of mistuned piezoelectric shunt systems and its estimation. Shock and Vibration 2016: 9739217 (18pp).

Berardengo M, Thomas O, Giraud-Audine C and Manzoni S (2016) Improved resistive shunt by means of negative capacitance: new circuit, performances and multi-mode control. Smart Materials and Structures 25: 075033 (23pp).

Caruso G (2001) A critical analysis of electric shunt circuits employed in piezoelectric passive vibration damping. Smart Materials and Structures 10: 1059-1068.

Cheng TH and Oh IK (2009) A current-flowing electromagnetic shunt damper for multi-mode vibration control of cantilever beams. Smart Materials and Structures 18: 095036 (10pp).

Collet M and Cunefare KA (2008) Modal synthesis and dynamical condensation methods for accurate piezoelectric systems impedance computation. Journal of Intelligent Material Systems and Structures 19: 1251-1269.

Den Hartog JP (1956) Mechanical Vibrations (4th edn). McGraw-Hill, New York. (Reprinted by Dover, New York, 1985) 
Fleming AJ (2004) Synthesis and Implementation of Sensor-less Shunt Controllers for Piezoelectric and Electromagnetic Vibration Control. PhD thesis, The University of Newcastle, Callaghan, Australia.

Fleming AJ and Moheimani SOR (2006) Inertial vibration control using a shunted electromagnetic transducer. IEEE/ASME Transactions on Mechatronics 11: 84-92.

Forward RL (1979) Electronic damping of vibrations in optical structures. Applied Optics 18: 690-697.

Gonzalez-Buelga A, Clare LR, Neild SA, Jiang JZ and Inman DJ (2015) An electromagnetic ineter-based vibration supression device. Smart Materials and Structures 24: 055015 (10pp).

Hagood NW and von Flotow A (1991) Damping of structural vibrations with piezoelectric materials and passive electrical networks. Journal of Sound and Vibration 146: 243-268.

Høgsberg J and Krenk S (2012) Balanced calibration of resonant shunt cicuits for piezoelectric vibration control. Journal of Intelligent Material Systems and Structures 23: 1937-1948.

Høgsberg J and Krenk S (2015) Balanced calibration of resonant piezoelectric RL shunts with quasi-static background flexibility correction. Journal of Sound and Vibration 341: 16-30.

Høgsberg J and Krenk S (2017) Calibration of piezoelectric RL shunts with explicit residual mode correction. Journal of Sound and Vibration 386: 65-81.

Høgsberg J (2019) A robust calibration procedure for multiple electromechanical shunt absorbers on a flexible structure. IX ECCOMAS Thematic Conference on Smart Structures and Materials (SMART 2019), A. Benjeddou, N. Mechbal and J.F. Deü (Eds).

Inoue T, Ishida Y and Sumi M (2008) Vibration suppression using electromagnetic resonant shunt damper. Journal of Vibration and Acoustics 130: 041003 (8pp).

Krenk S (2005) Frequency analysis of the tuned mass damper. Journal of Applied Mechanics 72: 936-942.

Krenk S and Høgsberg J (2013) Equal modal damping design for a family of resonant vibration control formats. Journal of Vibration and Control 19: 1294-1315.

Krenk S and Høgsberg J (2014) Tuned mass absorber on a flexible structure. Journal of Sound and Vibration 333: 1577-1595.

Krenk S and Høgsberg J (2016) Tuned resonant mass or inerter-based absorbers: unified calibration with quasi-dynamic flexibility and inertia correction. Proceedings of the Royal Society A - Mathematical, Physical and Engineering Sciences 472: 20150718 (23pp). DOI: 10.1098/rspa.2015.0718.

de Marneffe B and Preumont A (2008) Vibration damping with negative capacitance shunts: theory and experiment. Smart Materials and Structures 17: 035015 (9pp).

McDaid AJ and Mace BR (2013) A self-tuning electromagnetic vibration absorber with adaptive shunt electronics. Smart Materials and Structures 22: 105013 (9pp).

McGowan PE, Edighoffer HE and Wallace JW (1990) Deveoplment of an Experimental Space Station Model for Structural Research. NASA TM-102601, NASA Langley Research Center, Hampton, VA, USA. 
Moheimani SOR and Fleming AJ (2006) Piezoelectric transducers for vibration control and damping. Springer, London.

Niederberger D (2005) Smart Damping Materials using Shunt Control. PhD thesis, Swiss Federal Institute of Technology (ETH) Zurich, Zurich, Switzerland.

Niederberger D, Behrens S, Fleming AJ, Moheimani SOR and Morari M (2006) Adaptive electromagnetic shunt damping. IEEE/ASME Transactions on Mechatronics 11: 103-108.

Niu HP, Zhang XN, Xie SL and Wang PP (2009) A new electromagnetic shunt damping treatment and vibration control of beam structures. Smart Materials and Structures 18: 045009 (15pp).

Palomera-Arias R (2005) Passive electromagnetic damping device for motion control of building structures. PhD thesis, Massachusetts Institute of Technology, USA.

Palomera-Arias R, Connor JJ and Ochsendorf JA (2008) Feasibility study of passive electromagnetic damping systems. Journal of Structural Engineering 134: 164-170.

Porfiri M, Maurini C and Pouget J (2007) Identification of electromechanical modal parameters of linear piezoelectric structures Smart Materials and Structures 16: 323-331.

Preumont A (2011) Vibration Control of Active Structures. An Introduction, 3rd edition. Springer, Heidelberg.

Stabile A, Aglietti GS, Richardson G and Smet G (2017) Design and verification of a negative resistance electromagnetic shunt damper for spacecraft micro-vibration. Journal of Sound and Vibration 386: 38-49.

Scheidler JJ and Asnani VM (2017) Validated linear dynamic model of electrically-shunted magnetostrictive transducers with application to structural vibration control. Smart Materials and Structures 26: 035057 (17pp).

Tang XD, Liu YL, Cui W and Zuo L (2016) Analytical solutions to H-2 and H-infinity optimizations of resonant shunted electromagnetic tuned mass damper and vibration energy harvester. Journal of Vibration and Acoustics 138: 011018 (8pp).

Thomas O, Deü JF and Ducarne J (2009) Vibrations of an elastic structure with shunted piezoelectric patches: efficient finite element formulation and electromechanical coupling coefficients. International Journal for Numerical Methods in Engineering 80: 235-268.

Thomas O, Ducarne J and Deü JF (2012) Performance of piezoelectric shunts for vibration reduction. Smart Materials and Structures 21: 015008 (16pp).

Toftekær JF, Benjeddou A and Høgsberg J (2019) General numerical implementation of a new piezoelectric shunt tuning method based on the effective electromechanical coupling coefficient. Mechanics of Advanced Materials and Structures DOI: 10.1080/15376494.2018.1549297.

Toftekær JF and Høgsberg J (2020) Multi-mode piezoelectric shunt damping with residual mode correction by evaluation of modal charge and voltage. Journal of Intelligent Material Systems and Structures 31: 570-586.

Wu SY (1996) Piezoelectric shunts with a parallel R-L circuit for structural damping and vibration control. Proceedings of the Society of Photo-Optical Instrumentation Engineers (SPIE) 2720, San Diego, California, 259-269. 
Yamada K, Matsuhisa H, Utsuno H and Sawada K (2010) Optimum tuning of series and parallel LR circuits for passive vibration suppression using piezoelectric elements. Journal of Sound and Vibration 329: 5036-5057.

Yan B, Luo Y and Zhang X (2016) Structural multimode vibration absorbing with electromagnetic shunt damping. Journal of Vibration and Control 22: 1604-1617.

Yan B, Wang K, Hu Z, Wu C and Zhang X (2017) Shunt Damping Vibration Control Technology: A Review. Applied Sciences 7: 494 (31pp).

Zhu SY, Shen WA and Qian X (2013) Dynamic analogy between an electromagnetic shunt damper and a tuned mass damper. Smart Materials and Structures 22: 115018 (11pp).

Zhang XN, Niu HP and Yan B (2012) A novel multimode negative inductance negative resistance shunted electromagnetic damping and its application on a cantilever plate. Journal of Sound and Vibration 331: 2257-2271. 